\title{
Análise de Inadimplência em Dados de Faturamento Utilizando Rede Bayesiana Ingênua Aumentada em Árvore
}

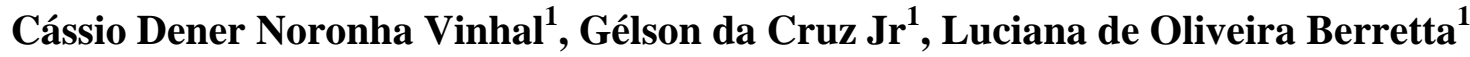 \\ ${ }^{1}$ Escola de Engenharia Elétrica e de Computação - UFG
} cassio@eee.ufg.br,gcruz@eee.ufg.br,lberretta@nepe.eee.ufg.br

\begin{abstract}
Resumo. O presente trabalho verifica a aplicabilidade de Classificadores Bayesianos em Bancos de Dados de faturamento de uma distribuidora de energia. $O$ intuito é encontrar padrões ou perfis em determinados grupos de consumo e estimar a quantidade de inadimplentes. O sistema computacional identifica padrões no histórico de cada cliente e projeta comportamentos prováveis. Utilizou-se o classificador Bayesiano Ingênuo Aumentado em Árvore em contraposição ao Bayesiano Ingênuo. A validação é feita através da comparação das taxas de acertos nas predições. As conclusões indicam uma abordagem adequada que oferece subsídios para se estabelecer políticas comerciais efetivas e reduzir a inadimplência.
\end{abstract}

\section{Introdução}

A competitividade entre as empresas tem aumentado muito e para se manterem no mercado e obter uma maior lucratividade, essas empresas buscam novas e sofisticadas alternativas tecnológicas fundamentadas na matemática, sistemas de informação e engenharia para a redução de custos e aumento de lucros. Os resultados esperados permitem aumentar a variedade de produtos e serviços ofertados, conquistar novos mercados, realizar um "marketing” mais elaborado, adotar estratégias para manter o cliente e evitar a inadimplência.

O investimento em tecnologias permite observar que os processos administrativos estão se tornando cada vez mais informatizados e permitem o acúmulo de dados sobre compras e vendas, clientes, entre outros. Os dados permitem constituir Bancos de Dados que são armazenados em "Sistemas Gerenciadores de Bancos de Dados”, compondo um grande histórico das transações das empresas e seus clientes. Entretanto dados produzidos e armazenados em larga escala muitas vezes não podem ser analisados por meio de métodos manuais tradicionais. Por outro lado, uma grande quantidade de dados pode vir a ser uma fonte de mais e melhores informações para a elaboração de políticas de negócios mais efetivas. Surge assim a necessidade de se explorar estes dados para extrair um conhecimento implícito, e. g., padrões ou regras importantes ali “escondidas” e que podem ser úteis para a tomada de decisões.

A utilização de técnicas como classificação, regras de associação, entre outras, tem aumentado muito. Nesse contexto o presente trabalho investiga modelos computacionais capazes de indicar a probabilidade de determinados consumidores se tornarem inadimplentes e fornecer medidas sobre a quantidade de inadimplentes, perfis de inadimplência dentro de grupos determinados, etc. Especificamente, um classificador 
Bayesiano Ingênuo Aumentado em Árvore é analisado em contraposição ao Bayesiano Ingênuo e os resultados obtidos são muito promissores (Berretta, 2005).

\section{Classificação}

Classificação é uma tarefa muito importante para a identificação de padrões e predições. Em geral, uma classificação é uma função que permite determinar, a partir de um grupo pré-definido de rótulos, uma classe específica, segundo instâncias descritas por um conjunto de atributos.

Em Han et al. (2001), a classificação de dados é um processo onde, no primeiro passo, é construído um modelo que descreve um conjunto de classes predeterminadas ou conceitos. Este modelo é construído pela análise das amostras (tuplas) do Banco de Dados, descritas pelos atributos. As amostras individuais, escolhidas aleatoriamente, formam um conjunto de treino e procedimentos de aprendizado podem ser utilizados.

Classificadores Bayesianos são classificadores estatísticos. Eles podem predizer a probabilidade de um membro de uma classe e a probabilidade de uma dada amostra pertencer a uma classe particular.

\section{Rede Bayesiana Ingênua}

A Rede Bayesiana Ingênua (Naïve-Bayes (NB)) é uma estrutura simples com nós classificados como nós pais de todos os outros nós, sendo que nenhuma outra conexão é permitida.

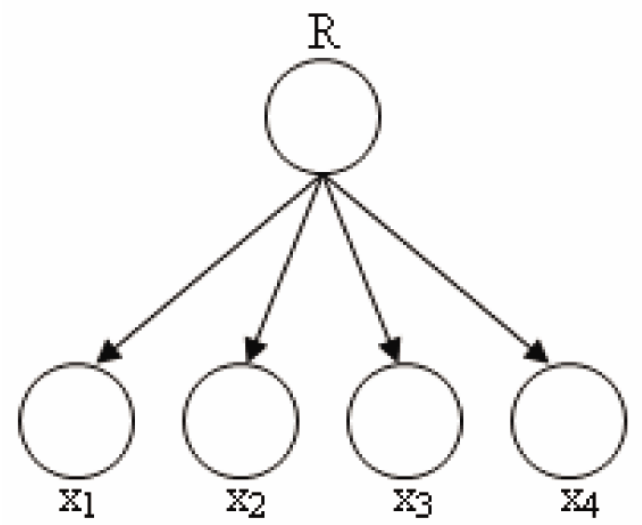

Figura 1 Exemplo de Rede Bayesiana Ingênua.

Segundo Mello (2001), a rede NB tem sido usada em classificadores há muitos anos, e possui duas vantagens sobre os outros classificadores: a) é de fácil construção e nenhum procedimento de aprendizagem é requerido b) o processo de classificação é muito eficiente computacionalmente, desde que ele assuma que todas as características são independentes das outras. Embora esta suposição possa ser problemática, a rede NB pode surpreender, apresentando-se superior a classificadores sofisticados onde as características não são fortemente combinadas.

O procedimento de construção de uma rede NB consiste basicamente em permitir que o nó de classificação seja o pai de todos os outros nós (nós filhos), não 
sendo permitida a conexão entre os filhos. Não é necessário métodos para se levantar a estrutura da rede. Neste trabalho será utilizada uma rede Bayesiana diferente, cujos resultados obtidos nos estudos de caso serão comparados aos de uma NB. Portanto, a seguir é descrito um algoritmo para levantamento da estrutura de uma rede.

\section{Algoritmo de Chow-Liu}

O algoritmo utilizado para construir a estrutura da rede desse trabalho é baseado em um método conhecido na literatura como Algoritmo de Chow-Liu (1968) devido ao trabalho pioneiro cuja idéia é comparar distribuições diferentes sobre duas variáveis, consideradas dependentes ou independes, consistentemente com o domínio em que são estimadas, a partir de bancos de dados.

Um grafo não-direcionado é formado quando iniciado por um grafo sem arcos, adicionando-se um arco entre dois nós com máxima entropia. Logo após, um arco com máxima entropia associada é adicionado, desde que não crie um ciclo no grafo. Este processo é repetido até que não seja possível adicionar arcos. O passo final consiste em associar direções aos arcos de maneira a formar uma árvore. Pearl (1988) divide o método em duas fases. Na primeira fase ocorre a geração da árvore ponderada máxima, que produz um grafo não-direcionado contendo, a relação das variáveis do problema. Na segunda fase ocorre a definição da direcionalidade dos arcos.

A primeira fase é descrita na forma de algoritmo com cinco passos:

1)Dada uma distribuição $\mathrm{P}(\mathrm{x})$, computam-se as distribuições conjuntas $\mathrm{P}\left(\mathrm{x}_{i}, \mathrm{x}^{j}\right)$ para todos os pares de variáveis;

2)Utilizando-se as distribuições calculadas no passo 1, calculam-se os pesos para todos os $n(n-1) / 2$ ramos da árvore, que devem ser ordenados por ordem de magnitude. Esses pesos são calculados pela equação da informação mútua, cujo desenvolvimento pode encontrado no trabalho de Pearl.

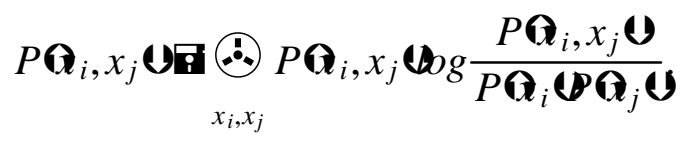

3)Associam-se os dois ramos de maior peso a árvore a ser construída.

4)O próximo ramo da lista, já ordenada, deve ser acrescentado à árvore, contanto que não seja criado um ciclo. Caso isto aconteça, este ramo deve ser destacado e o próximo deve ser selecionado.

5)Repete-se o passo 4 até que $n$ - 1 ramos tenham sido selecionados. Nesse ponto, o esqueleto da árvore está construído.

A segunda fase direciona os arcos, calculando a projeção de probabilidade de $\mathrm{P}$ " (x) sobre a distribuição $\mathrm{P}(\mathrm{x})$, selecionando um nó arbitrário para a raiz e formando o produto dado pela equação: 


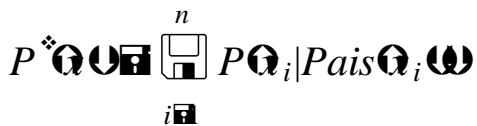

Sua complexidade é $\mathrm{O}\left(\mathrm{n}^{2}\right)$ e se utiliza apenas de comparações de pesos dos ramos.

\section{Rede Bayesiana Ingênua Aumentada em Árvore}

Uma Rede Bayesiana Ingênua Aumentada em Árvore (Tree Augmented Nä̈ve-Bayes (TAN)) é uma estrutura que tem nós classificados como nós pais de todos os outros nós e permite conexões entre os nós filhos.

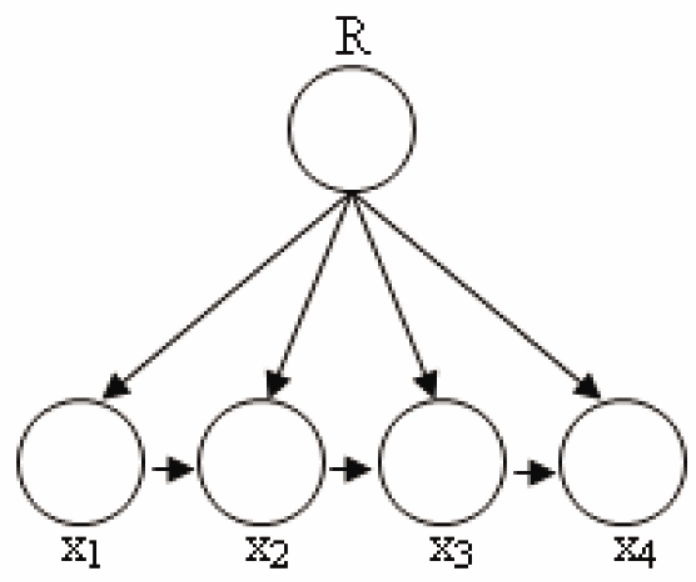

Figura 2 Exemplo de Rede Bayesiana Ingênua Aumentada em Árvore

Permitindo $\mathrm{X}=\{\mathrm{x} 1, \ldots, \mathrm{X} n, \mathrm{R}\}$ representar o conjunto de nós (onde $\mathrm{R}$ é o nó de classificação) dos dados, o algoritmo para aprendizagem da classificação TAN aprende uma árvore estruturada sobre $X \mid\{R\}$, usando mútuos testes de informações. Ele, então, adiciona uma ligação do nó de classificação para cada característica do nó, à maneira como constrói uma rede Bayesiana Ingênua. Uma estrutura TAN simples é mostrada na Figura 2. Note que as características X 1, x 2, х з e x 4 formam uma árvore (CHENG et al., 2000).

Um procedimento de aprendizagem pode ser descrito como se segue:

1)Tomam-se o conjunto preparado e $X \mid\{R\}$ como entradas;

2)Chama-se o algoritmo Chow-Liu modificado e substitui-se todo o teste de informação

mútua $I\left(x_{i}, X_{j}\right)$ por um teste de informação condicional $\left.I\left(x_{i}, x^{j}\right) \mid\{R\}\right)$;

3)Adiciona-se $\mathrm{R}$ como pai de todos $x$ i, onde $1 \hat{\vee} i \hat{\vee} n$;

4)Aprendem-se os parâmetros e produz-se a TAN.

A TAN pode ser descrita da seguinte forma: 


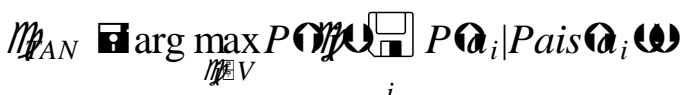

\section{Modelo de Predição}

Com o objetivo de realizar a predição de inadimplentes dos dados de faturamento de uma distribuidora de energia, foi desenvolvido um modelo que se utiliza de um classificador. Os classificadores podem predizer a probabilidade de um membro de uma classe e a probabilidade de uma dada amostra pertencer a uma classe particular.

Como elemento classificador foi utilizado uma Rede Bayesiana Ingênua Aumentada em Árvore (TAN). O algoritmo para aprendizagem da classificação TAN aprende uma árvore estruturada sobre $\mathrm{X} \mid\{\mathrm{R}\}$ como já descrito, usando testes de informações mútuas.

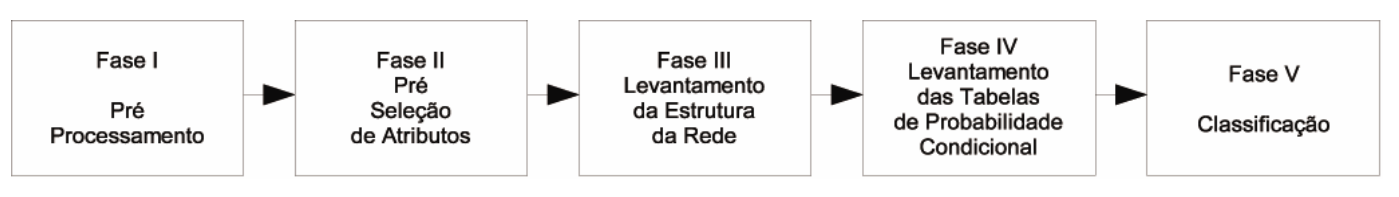

Figura 3 Fases do Modelo

Com o intuito de facilitar a compreensão do modelo, este pode ser dividido em cinco etapas. A primeira etapa é o pré-processamento, responsável pela limpeza, desnormalização e discretização dos dados. A segunda etapa é a pré-seleção dos atributos, na qual a relevância dos atributos é analisada. A terceira etapa é o levantamento da estrutura da rede. A quarta é o levantamento das Tabelas de Probabilidade Condicional, que são as informações estatísticas dos dados. E finalmente, a fase de classificação, que faz a previsão dos possíveis inadimplentes.

\subsection{Pré-Processamento}

Na maioria das vezes, os dados não se encontram em um formato adequado, tornandose necessário que os mesmos sejam tratados, a fim de permitir uma melhor aplicação do algoritmo de classificação. A etapa do Pré-Processamento é responsável por preparar esses dados para a análise.

De modo a promover a exatidão, a eficiência e a escalabilidade no processo de classificação, alguns passos devem ser seguidos, dentre os quais se destacam:

- Limpeza de Dados. Este procedimento, segundo Han et al. (2000), refere-se à remoção ou redução do "ruído" e ao tratamento dos dados ausentes. Isto pode ajudar a reduzir a confusão durante o aprendizado. Um aspecto importante desta fase é o tratamento dos valores ausentes;

- Desnormalização. O modelo de dados normalizado na 3FN (Terceira Forma Normal) pode requerer um maior número de junções para processar uma consulta e isso pode ser otimizado. A desnormalização retorna à 2FN (Segunda Forma Normal) ou à 1FN (Primeira Forma Normal), dependendo do caso;

- Discretização. A maioria dos algoritmos de aprendizagem de redes Bayesianas trabalha com variáveis categóricas (discretas não-ordenáveis), pois alguns 
campos podem oferecer melhor desempenho na classificação se forem tratados como valores discretos. A técnica de discretização utilizada neste trabalho foi a dos K-Intervalos Proporcionais para Classificadores Bayesianos (Yang, 2003).

\subsection{Pré-Seleção de Atributos}

Na etapa de Pré-seleção dos Atributos é feita uma análise de relevância, pois muitos dos atributos dos dados podem ser irrelevantes para a tarefa de classificação. Além disso, outros atributos podem ser redundantes. Incluir atributos sem necessidade pode tornar 0 processo lento e possivelmente induzir a erro na etapa de treinamento.

\subsection{Levantamento da Estrutura da Rede}

Após as fases de pré-processamento e de pré-seleção dos atributos, faz-se necessário o levantamento da estrutura da rede. A estrutura da rede é uma representação abstrata do conhecimento do domínio, ou seja, a estrutura causal entre os processos do domínio.

Um modelo faz o levantamento da estrutura da rede de forma automática. O método utilizado para redes TAN já foi discutido (Seções 4 e 5). Ele toma uma distribuição de probabilidade $\mathrm{P}$ como entrada e constrói uma estrutura de árvore como saída. Para redes NB não é preciso levantar a estrutura, como explicado na Seção 3.

\subsection{Levantamento da Tabela de Probabilidades Condicionais}

Uma vez definida a estrutura da rede, é preciso especificar as probabilidades condicionais para os nós que participam diretamente das relações de dependência. Cada nó possui uma tabela de probabilidade condicional que quantifica a influência que os nós pais têm sobre cada nó filho. Essa construção consiste em levantar a probabilidade de cada nó $\mathrm{X}_{i}$, dados seus pais (Pais $\left.\left(\mathrm{X}_{i}\right)\right)$ - $\mathrm{P}\left(\mathrm{X}_{i} \mid \mathrm{Pais}\left(\mathrm{X}_{i}\right)\right)$.

\subsection{Classificação}

As redes Bayesianas escolhidas para realizar o processo de classificação neste trabalho permitem encontrar probabilidades para todas as classes. A classe que apresentar a maior probabilidade é a escolhida como a classe do elemento.

\section{Resultados}

Os estudos de caso foram produzidos para grupos com predomínio de clientes da classe residencial (100\% Residencial; 90\% a 100\% Residencial; 80\% a 90\% Residencial; 70\% a $80 \%$ Residencial). Observou-se que em classes mais uniformes (e.g., 100\% residencial) a redes NB e TAN apresentam desempenhos muito parecidos.

Cada grupo foi subdividido em bairros, com 5 faixas de consumo (abaixo de $100 \mathrm{kWh} / \mathrm{mês} ; 100 \mathrm{kWh} / \mathrm{mês}$ a $200 \mathrm{kWh} / \mathrm{mês} ; 200 \mathrm{kWh} / \mathrm{mês}$ a $300 \mathrm{kWh} / \mathrm{mês} ; 300 \mathrm{kWh} / \mathrm{mês}$ a $500 \mathrm{kWh} / \mathrm{mês} ; 500 \mathrm{kWh} / \mathrm{mês}$ a $800 \mathrm{kWh} / \mathrm{mês}$ ) como pode ser visto na Tabela 1 . As primeiras faixas de consumo tendem a apresentar clientes de mais baixo consumo e também de menor poder aquisitivo.

Cada faixa direciona um experimento feito em 2 etapas, treinamento e classificação. Na etapa de treinamento, foram utilizadas amostras dos dados referentes aos meses de outubro, novembro e dezembro de 2002, e na etapa de teste, foram 
utilizados dados dos meses de janeiro, fevereiro e março de 2003. Não foram utilizados dados do período de racionamento.

Em se tratando da predição de inadimplência, o erro foi medido em relação a valores históricos reais do Banco de Dados de uma distribuidora de energia. O desempenho das redes TAN é melhor que aquele das redes NB em todas as faixas do exemplo da Tabela 1, e.g., Bairro Jd. América. No desempenho global, considerando todos os estudos de caso, observa-se novamente que as redes TAN se sobressaem em relação às redes NB, conforme mostra a Tabela 2.

Tabela 1 Resultados - Bairro Jardim América

\begin{tabular}{|c|c|c|c|}
\hline Faixa & $\begin{array}{c}\text { Amostr } \\
\mathbf{a}\end{array}$ & \% Erro (NB) & \% Erro (TAN) \\
\hline 1 & 12171 & 10,62 & 6,65 \\
\hline 2 & 15587 & 2,09 & 1,90 \\
\hline 3 & 7622 & 1,70 & 1,00 \\
\hline 4 & 4948 & 1,01 & 0,76 \\
\hline 5 & 1142 & 2,54 & 0,70 \\
\hline
\end{tabular}

Tabela 2 Desempenho da TAN em relação à NB

\begin{tabular}{|c|c|c|c|}
\hline & Ganhou & Perdeu & Empatou \\
\hline $\mathrm{N}^{\circ}$ Casos & 33 & 12 & 5 \\
\hline Percentual & $66 \%$ & $24 \%$ & $10 \%$ \\
\hline
\end{tabular}

\section{Conclusão}

Após o desenvolvimento dos experimentos e analisando a abordagem proposta, pode-se concluir que, apesar da ocorrência de erros pequenos em sua maioria, os resultados são promissores. Para gerar resultados significativos, as amostras devem possuir um quantitativo mínimo para cada classe, a fim de evitar exclusividade na previsão de alguma classe.

O classificador TAN apresentou taxas de erro elevadas nas previsões realizadas para faixas de baixo consumo de energia (Tabela 1) e nas quais se verificaram taxas mais altas de inadimplência. Acredita-se que a escolha de atributos que possam refletir o perfil sócio-econômico em cada faixa, possa contribuir para uma diminuição da taxa de erros quando da caracterização de inadimplentes.

Os resultados também apontam para o desenvolvimento de novas políticas que definam inadimplência de uma forma mais flexível, permitindo a consideração de atrasos no pagamento por motivos diversos, flexibilidade de datas de pagamento, dentre outros, conforme apontado por especialistas da área de comercialização de energia.

Acredita-se que melhorias no modelo podem ser feitas em consideração a tais ponderações e que resultarão na melhoria das predições. Finalmente, a hipótese de o 
classificador ser capaz de prever o comportamento dos consumidores pôde ser confirmada, e a TAN se mostrou mais precisa que a NB.

\section{Referências}

BERRETTA, L.O. (2005), “Análise de Inadimplência em Dados de Faturamento Utilizando Rede Bayesiana Ingênua Aumentada em Árvore”, Dissertação de Mestrado, EEEC - UFG, Goiânia, Brasil.

CHOW, C., LIU, C. (1968), “Approximating Discrete Probability Distributions with Dependence Trees”. IEEE Transactions on Information Theory, vol.14-3, 462- 467, USA.

CHENG, J.; GREINER, R. (2000) “Comparing Bayesian Network Classifiers”. Alberta, CA: University of Alberta, 2000.

HAN, J.; KAMBER, M. (2000) “Data Mining, Concepts and Techniques”. USA, Morgan Kaufmann,

MELLO, L. C. (2001) "Uma revisão de abordagens genético-difusas para descoberta de conhecimento em banco de dados”. Porto Alegre, RS: Universidade Federal do Rio Grande do Sul - UFRS.

PEARL, J. (1988) "Probabilistic Reasoning in Intelligent Systems: Networks of Plausible Inference”. USA, Morgan Kaufmann.

YANG, Y. (2003) "Discretization for Naïve-Bayes Learning”. [S.l.]: School of Computer Science and Software Engineering of Monash University. 